EPJ Web of Conferences 110,01077 (2016)

DOI: $10.1051 /$ epjconf/201611001077

(C) Owned by the authors, published by EDP Sciences, 2016

\title{
ABILITY OF FULLERENE TO ACCUMULATE HYDROGEN
}

\author{
Mikhail A Bubenchikov ${ }^{1}$, Alexey M Bubenchikov ${ }^{1}$, Olesya V Usenko ${ }^{1,}$, , Valentina B Tsyrenova ${ }^{2}$ and \\ Sanghi O Budaev ${ }^{2}$ \\ ${ }^{1}$ National Research Tomsk State University, 634050, 36 Lenin av., Tomsk, Russia \\ ${ }^{2}$ Buryat State University, 670000, 24a Smolina st., Ulan-Ude, Russia
}

\begin{abstract}
In the present paper, using a modification of the LJ-potential and the continuum approach, we define $\mathrm{C}_{60}-\mathrm{H}_{2}(\mathrm{He})$ potentials, as well as interaction energy of two fullerene particles. The proposed approach allows to calculate interactions between carbon structures of any character (wavy graphenes, nanotubes, etc.). The obtained results allowed to localize global sorption zones both inside the particle and on the outer surface of the fullerene.
\end{abstract}

\section{Introduction}

Fullerenes are used in medicine [1, 2], in water purification [3], as biosensors [4] and in many other spheres. In the present paper, $\mathrm{C}_{60}$ molecules, along with the other components of carbon black, are considered from the point of view of forming advanced carbon membranes for gas separation.

Since the discovery of the fullerene in 1985 [5], numerous works have been dedicated to studying the synthesis of these particles. A fullerene is obtained, for example, by means of maintaining the carbon-helium plasma with the help of direct or alternating current [6] or from carbon black [7]. Alternatively, a fullerene molecule can be formed from primary clusters of $\mathrm{C}_{2}$, which are then joined together to form a series of intermediates [8]. Recently, it has become possible to receive new graphite- and diamond-like structures from $\mathrm{C}_{60}$, as a result of decomposition of $\mathrm{C}_{60}$ under the influence of irradiation or helium plasma [9].

Currently, the Lennard-Jones potential (LJ-potential) is used to describe the interaction of a fullerene with different particles: to comprehensively study fullerene motion in open carbon nanocones [10], to describe the dynamics of the $\mathrm{C}_{60}$-graphite collision [11] to study encapsulation of $\mathrm{C}_{60}$ in single-walled carbon tubes [12], to determine the dynamic factors of fullerene nanofluids [13], and also a LJ-potential additive is used to generate both translational and anisotropic-rotational motion of each fullerene particle [14]. The use of the LJ-potential and the Coulomb potential allows to analyze movement of fullerene structures within an ensemble of particles of the same kind, as well as movement of potassium ions inside a fullerene "sphere" [15].

Using the Monte Carlo computer simulation we studied fullerene adsorption of such gases as ethylene [16], nitrogen [17], argon and carbon dioxide [18], as well as adsorption of hydrogen onto a neutral fullerene [19] and helium onto $\mathrm{C}_{60}$ and $\mathrm{C}_{70}$ [20]. The Monte Carlo simulation is also used to study the collective behaviour of particles consisting of two fullerene spheres and two mesogenic fragments rigidly attached to them [21].

\section{${ }^{a}$ Corresponding author : usenko.olesya@yandex.ru}


With the help of molecular dynamics methods it is possible to investigate thermodynamic properties of $\mathrm{H}_{2}$ and $\left(\mathrm{H}_{2}\right)_{2}$ inside of $\mathrm{C}_{60}$ [22], to study the interaction of fullerenes with a wavy grapheme surface [23], to study the dynamics of a fullerene molecule fluctuations in a single-walled carbon nanotube [24], to perform calculations of the strong coupling of sixty carbon atoms in a helium atmosphere [25], to study mechanical properties of hybrid structures composed of blocks of various shapes [26].

On the basis of the surface continuum for the distribution of carbon atoms energy along the crystal layer, fullerene-fullerene and fullerene-graphene interactions are thoroughly investigated [27].

\section{Methods}

\subsection{Power centres - interaction energy sources}

The continuum model of the interaction of nanoparticles with molecules was developed in [28-35]. The normalized LJ-potential has the form:

$$
\Phi(\rho)=\frac{1}{\rho^{12}}-\frac{1}{\rho^{6}},
$$

where $\rho=\sqrt{\left(x-x^{\prime}\right)^{2}+\left(y-y^{\prime}\right)^{2}+\left(z-z^{\prime}\right)^{2}}, x, y, z, x^{\prime}, y^{\prime}, z^{\prime}$, are the Cartesian coordinates of the interacting molecules' centres. Equation (1) can be modified:

$$
\Phi_{1}(\rho)=\frac{1}{\rho} \operatorname{th}\left(\frac{1-\rho^{6}}{\rho^{11}}\right)
$$

Then at $\rho \rightarrow 0 \quad \Phi_{1}(\rho) \rightarrow \infty$ in the same way as $\frac{1}{\rho}$.

In the figure 1 are shown comparing graphs of functions $\Phi(\rho)$ and $\Phi_{1}(\rho)$.
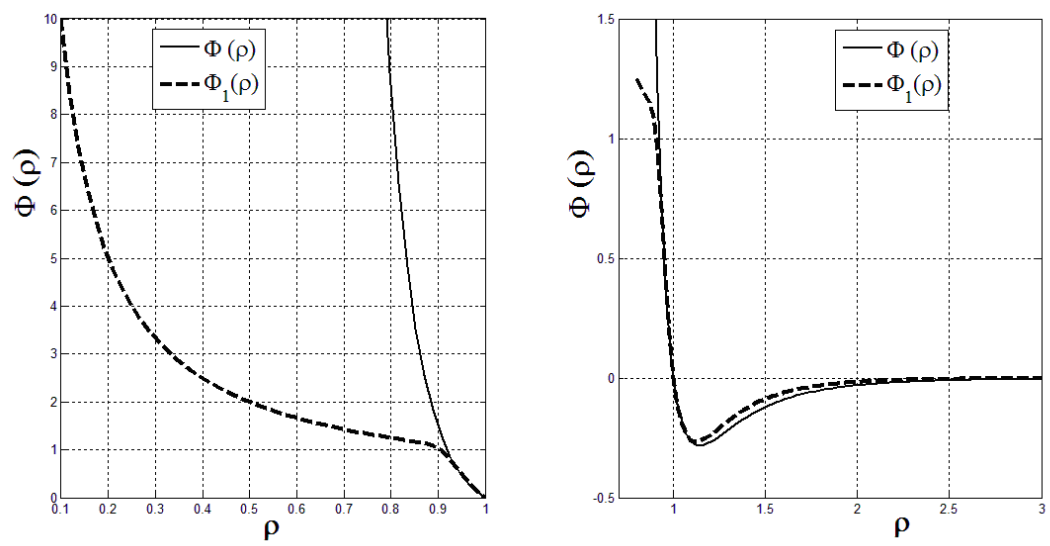

Figure 1. Comparing graphs of functions $\Phi(\rho)$ and $\Phi_{1}(\rho)$.

This modification of the Lennard-Jones potential has the integrable singularity at the origin.

\subsection{Modified LJ-potential}


Instead of (2) in the dimensional form we can write:

$$
\Pi_{1}(r)=4 \varepsilon\left(\frac{\sigma}{r}\right) \operatorname{th}\left[\left(\frac{\sigma}{r}\right)^{11}-\left(\frac{\sigma}{r}\right)^{5}\right]
$$

Here $\sigma$ is the radius at which the potential (3) vanishes; $\varepsilon$ is the depth of the potential well.

The values of the constants $\varepsilon$ and $\sigma$, included in the Lennard-Jones potential, for the homogeneous pairs of substances used in the work are given in the table 1 :

Table 1.

\begin{tabular}{|c|c|c|}
\hline Interacting molecules & $\begin{array}{c}\text { Relative depth of the } \\
\text { potential well (K) }\end{array}$ & $\begin{array}{c}\text { Influence radius of the } \\
\text { interaction potential (nm) }\end{array}$ \\
\hline $\mathrm{H}_{2}-\mathrm{H}_{2}$ & $\varepsilon / k=34$ & $\sigma=0,29$ \\
\hline $\mathrm{He}-\mathrm{He}$ & $\varepsilon / k=10,2$ & $\sigma=0,228$ \\
\hline $\mathrm{C}-\mathrm{C}$ & $\varepsilon / k=51,2$ & $\sigma=0,335$ \\
\hline
\end{tabular}

If the system under study consists of heterogeneous molecules (atoms), for the parameters $\varepsilon$ and $\sigma$ the following averaging rules (the Lorentz-Berthelot mixing rules) are observed:

$$
\sigma_{C_{2}}=\frac{\sigma_{C C}+\sigma_{H_{2} H_{2}}}{2}, \quad \varepsilon_{C H_{2}}=\left(\varepsilon_{C C} \cdot \varepsilon_{H_{2} H_{2}}\right)^{1 / 2}
$$

\subsection{Integration in case of interaction between simple molecules and a fullerene particle}

Within the scopes of continuum description, we assume that the intermolecular interaction energy is not localized at the corners of the crystal lattice but is uniformly distributed across the entire surface of the spherical fullerene.

Let $\bar{r}=(x, y, z)$ and $\bar{r}^{\prime}=\left(x^{\prime}, y^{\prime}, z^{\prime}\right)$ be the radius vectors of the interacting molecules. Then the distance between them in Cartesian coordinates will be:

$$
\left|\bar{r}-\bar{r}^{\prime}\right|=\sqrt{\left(x-x^{\prime}\right)^{2}+\left(y-y^{\prime}\right)^{2}+\left(z-z^{\prime}\right)^{2}} .
$$

The surface of the fullerene particle will be simulated by a sphere with uniformly distributed sources of energy on its surface; in addition we assume that $\bar{r}^{\prime}$ determines the position of the point on the sphere. Taking the relations of the Cartesian and spherical coordinates into account

$$
x=r \sin \theta \cos \varphi, \quad y=r \sin \theta \sin \varphi, \quad z=r \cos \theta
$$

instead of (4) we obtain:

$$
\left|\bar{r}-\bar{r}^{\prime}\right|=\sqrt{r^{2}+r^{\prime 2}-2 r r^{\prime}\left(\sin \theta \sin \theta^{\prime} \cos \left(\varphi-\varphi^{\prime}\right)+\cos \theta \cos \theta^{\prime}\right)} .
$$

The radius vector $\bar{r}$ will mark the position of the test molecule, with the help of which we will define the level of interaction with the particle. In other words, the point given by this vector can be 
considered the point of the observer. Since the spherical particle has a central symmetry, then, without restricting the generality, we can set $\theta=0$, and the expression (6) will be significantly simplified:

$$
|\bar{r}-\bar{r}|=\sqrt{r^{2}+r^{\prime 2}-2 r r^{\prime} \cos \theta^{\prime}} .
$$

Replacing in (3) the distance $r$ by the obtained expression, we integrate the interaction potential over the surface of the fullerene particle:

$$
U(r)=q \int_{s} \Pi_{1}\left(\sqrt{r^{2}+r^{\prime 2}-2 r r^{\prime} \cos \theta^{\prime}}\right) d s^{\prime} .
$$

Here $q$ is the density of carbon atoms distribution on the particle surface; $d s^{\prime}=H_{\varphi} d \varphi^{\prime} H_{\theta} d \theta^{\prime}$ is the area element on the particle surface; $H_{\varphi}=r^{\prime} \sin \theta^{\prime}, H_{\theta}=r^{\prime}$ are Lamé coefficients. As the angle $\varphi$ is not included in expression (7), and therefore in the integrand expression in (8), the integration of this parameter can be carried out analytically:

$$
U(r)=8 \pi \varepsilon \mathrm{a}^{2} q \int_{0}^{\pi}\left(\frac{\sigma}{\xi}\right) \operatorname{th}\left[\left(\frac{\sigma}{\xi}\right)^{11}-\left(\frac{\sigma}{\xi}\right)^{5}\right] \sin \theta^{\prime} d \theta^{\prime},
$$

where, in this case $\xi=\sqrt{r^{2}+a^{2}-2 a r \cos \theta^{\prime}}$, and a is the radius of the fullerene.

Integration in (9) was conducted using the trapezoidal rule for the elementary area over the $\operatorname{corner} \theta$. Figure 3 shows the calculation results for the energy of interaction between the fullerene and $\mathrm{He}$ and $\mathrm{H}_{2}$.

\subsection{Interaction energy of two fullerene particles}

In the figure 2, $O O_{1}=l$ is the distance between the centres of two fullerene spheres. A fragment of the particle, highlighted by two central sections, the angle between which is $\varphi$ is shown on the left. One section is in the plane of the sheet, the other passes through a point $M$ on the sphere. Deviation of the radius $O M$ from the vertical is determined by the angle $\theta$.

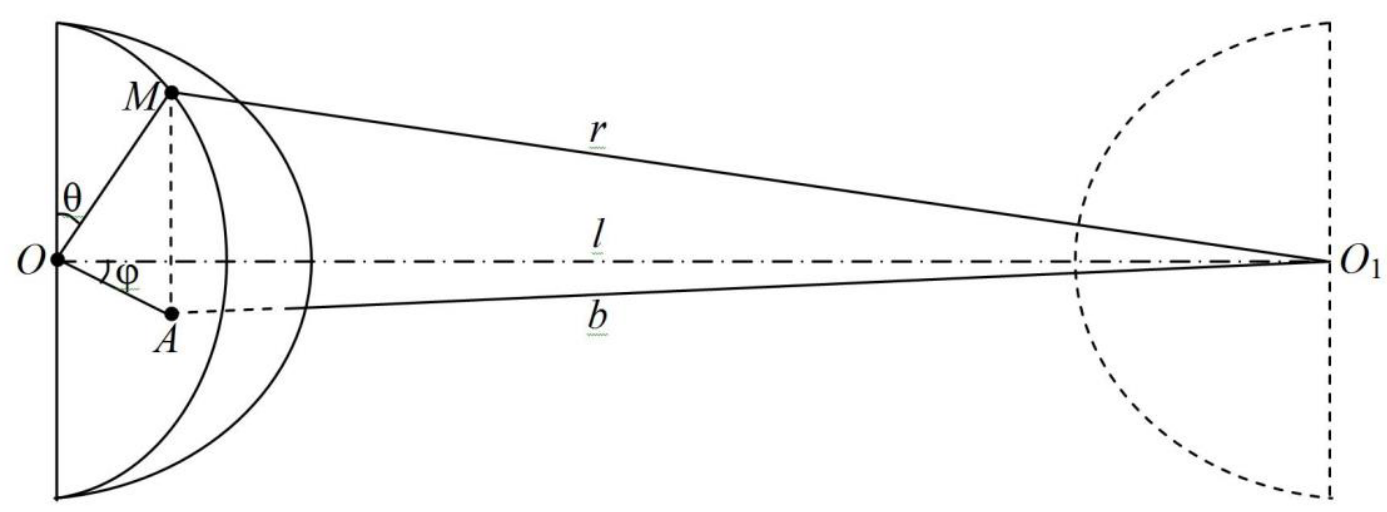

Figure 2. Determining interaction energy of two particles. 
Considering the triangle $O O_{1} A$ and using the cosine theorem, we get:

$$
b^{2}=a^{2} \sin ^{2} \theta+l^{2}-2 l a \sin \theta \cdot \cos \varphi .
$$

Since $O O_{1} A$ is the horizontal plane and $M A$ is height dropped onto this plane, the triangle $A M O_{1}$ is rectangular. Solving it, we can determine $M O_{1}=r$ :

$$
r=\sqrt{a^{2} \cos ^{2} \theta+b^{2}}
$$

Then in view of (10), we obtain

$$
r=\sqrt{a^{2}+l^{2}-2 l a \sin \theta \cdot \cos \varphi} .
$$

In fact, formula (12) determines the distance between the carbon atom located at the point $M$ on the surface of the fullerene particles on the left and the centre of the right particle. Moreover, the interaction potential $U(r)$ for this pair of objects is already known. Thus, the interaction energy of two fullerene particles is determined by the surface integral of $U(r)$ taken over the surface of the left particle on which carbon atoms have the same distribution density $q$ as on the right particle:

$$
\begin{array}{r}
U_{p}(l)=q \iint_{s} U(r) d s=q \int_{0}^{\pi} \int_{0}^{2 \pi} U(r) H_{\varphi} H_{\theta} d \varphi d \theta= \\
=q a^{2} \int_{0}^{\pi} \int_{0}^{2 \pi} U\left(\sqrt{a^{2}+l^{2}-2 l a \sin \theta \cdot \cos \varphi}\right) \sin \theta d \varphi d \theta .
\end{array}
$$

\section{Results and discussion}

Double integration in (13) can also be conducted using the trapezoidal rule. The integration results are shown in figure 4 . The distributions shown in figure 3 fix two sorption wells, as well as a wide energy barrier at the surface of the particle. The deep well in the centre of the fullerene particle provides a possibility of finding hydrogen molecules in some metastable state which is characterized by low mobility of molecules and their fairly close packing (the state close to the liquid phase, which provides intensive heat transfer through the graphene layer). A small well on the outer side of the fullerene indicates the ability of low-energy $\mathrm{H}_{2}$ molecules to be adsorbed in the near-surface layer of the particle. At $3.5 \mathrm{~nm}<\mathrm{r}<0.6 \mathrm{~nm}$ the energy of interaction between the molecule and the fullerene is positive, and the field of the Van der Waals forces produces a barrier (repellent) effect on the molecules of the area. Thus, low-energy molecules can not come to the fullerene any closer than at the distance of about $0.25 \mathrm{~nm}(0.7 \mathrm{a})$. At the same time, as seen from figure 4 , the surface of a $\mathrm{C}_{60}$ molecule can come to the same object at a distance two times shorter. However, the calculations show that fullerenes can not be closely packed. The considerably wide sorption well, obtained in the case of fullerene-fullerene interaction (figure 4), indicates the ability of the particles to be attracted to each other. In addition, the most probable distance between the nanoparticles surfaces is $0.25 \mathrm{~nm}$. 


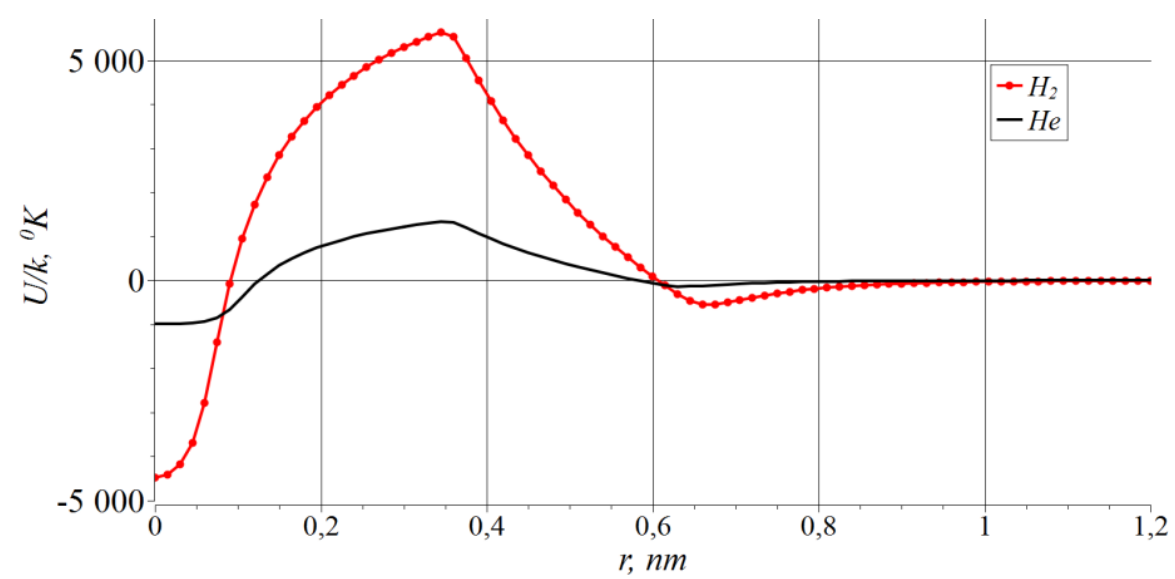

Figure 3. Distribution of interaction potentials of hydrogen and helium atoms and fullerene (inside and outside particle), $k$ - Boltzmann constant.

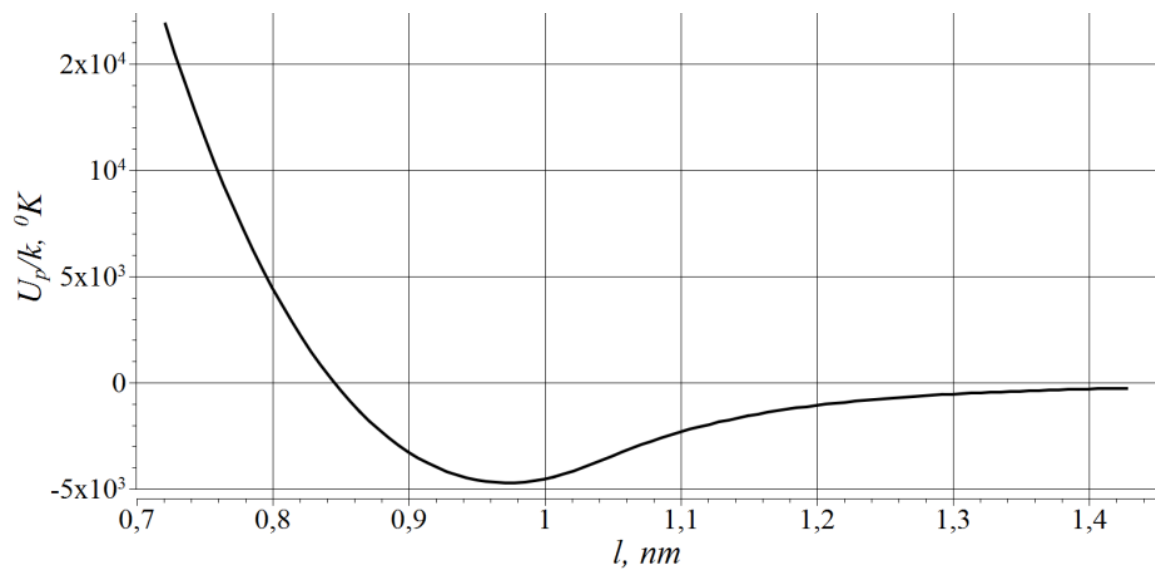

Figure 4. Interaction energy of two fullerene particles depending on distance between their centres, $k$ Boltzmann constant.

\section{Conclusion}

The potentials of intermolecular interactions $\mathrm{C}_{60}-\mathrm{H}_{2}, \mathrm{C}_{60}-\mathrm{He}$, as well as the potential $\mathrm{C}_{60}-\mathrm{C}_{60}$ were found. Due to the symmetry of the fullerene particle, the energy of interaction between the fullerene and the simple molecule $\left(\mathrm{He}\right.$ or $\left.\mathrm{H}_{2}\right)$ is determined by integrating the modified LJ-potential over one of the angles, using the spherical coordinate system. The potential $\mathrm{C}_{60}-\mathrm{C}_{60}$ is determined, as a final result, by a three-time integration over the angular coordinates of fullerene spheres. The sorption zones, characteristic for the fullerene, were determined. The most probable distance, which determines relative positioning of fullerene particles, was found.

\section{Acknowledgment}

This paper was written within the frame of Competitiveness Improvement Program of Tomsk State University. 


\section{References}

1. J.J. Ryan, H.R. Bateman, A. Stover, G. Gomez, S.K. Norton, W. Zhao, L.B. Schwartz, R. Lenk, and C.L. Kepley, J Immunol., 179 (2007)

2. R. Yin, M. Wang, Y.Y. Huang, H.C. Huang, P. Avci, L.Y. Chiang, and M.R. Hamblin, Nanomedicine, 10 (2014)

3. S. Chae, E.M. Hotze, and M.R. Wiesner, Micro and Nano Technologies; William Andrew Publishing: Oxford, 2014; pp. 329-338.

4. S. Afreen, K. Muthoosamy, S. Manickam, and U. Hashim, Biosens. Bioelectron., 63 (2015)

5. H.W. Kroto, J.R. Heath, S.C. O'Brien, R.F. Curl and R.E. Smalley, Nature, 318 (1985)

6. N.V. Bulina, V.A. Lopatin, P.V. Novikov, N.G. Vnukova, G.N. Churilov and W. Krätschmer, Fullerene synthesis in helium flow at atmospheric pressure, NATO Advanced Research Workshop on Hydrogen Materials Science and Chemistry of Carbon Nanomaterials, Sevastopol, Ukraine, September 05-11, 2005; NATO Security through Science Series A: Chemistry and Biology: Springer Netherlands, 2007; pp. 269-274.

7. A. Rotundi, F.J.M.Rietmeijer, and J. Borg, Springer Netherlands, 6 (2006)

8. A. Chuvilin, U. Kaiser, E. Bichoutskaia, N.A. Besley and A.N. Khlobystov, Nature Chem., 2 (2010)

9. E. Y. Kolyadina, L. A. Matveeva, P. L. Neluba, and V.V. Shlapatskaya, Mat.wiss.u.Werkstofftech., 44 (2013)

10. R. Ansari and Sadeghi F, Physica E Low Dimens Syst. Nanostruct., 69 (2015)

11. Z. Man, Z. Pan, Y. Ho, and W. Zhu, Nucl instrum meth B, 153 (1999)

12. H. Duan, X. Gao, G. Fu and J. Li, Phys. Lett. A, 375 (2011)

13. S. P. Tewari, G. Dhingra and P. Silotia, Int. J. Mod Phys. B, 24 (2010)

14. A. Piątek, A. Dawid and Z. Gburski, Spectrochim. Acta A, 79 (2011)

15. A. Piatek, A. Dawid and Z. Gburski, J. Mol. Struct., 887 (2008)

16. J.M.D. Tascón and E.J. Bottani, Carbon, 42 (2004)

17. A. Martínez-Alonso, J.M.D. Tascón and E.J. Bottani, Langmuir, 16 (2000)

18. A. Martínez-Alonso, J.M.D. Tascón and E.J. Bottani, J. Phys. Chem. B, 105 (2001)

19. A. Kaiser, C. Leidlmair, P.Bartl, S. Zöttl, S. Denifl, A. Mauracher, M. Probst, P. Scheier and O.

Echt, J. Chem. Phys., 138, 074311 (2013)

20. C. Leidlmair, Y. Wang, P. Bartl, H. Schöbel, S. Denifl, M. Probst, M. Alcamí, F. Martín, H. Zettergren, K. Hansen, O. Echt, and P. Scheier, Phys. Rev. Lett., 108, 076101 (2012)

21. A. Sazonovas, S. Orlandi, M. C. Ricci, Zannoni and E. Gorecka, Chem. Phys. Lett., 430 (2006)

22. G.E. Lopez, Phys. Lett. A, 376 (2012)

23. S.-Y. Kim, H.J. Hwang and J.W. Kang, Phys. Lett. A, 377 (2013)

24. J.W. Kang and H.J. Hwang. Comp. Mater. Sci., 50 (2010)

25. I. László, J. Mol. Struc-Theochem., 463 (1999)

26. Y. Zheng, L. Xu, Z. Fan, N. Wei, Y. Lu and Z. Huang, Curr. Nanosci., 8 (2012)

27. H. Guerin, J. Chim. Phys. PCB, 95 (1998)

28. A.M. Bubenchikov, M.A. Bubenchikov, A.I. Potekaev, E.E. Libin and Y.P. Khudobina, Izvestiya vuzov. Physics, 58, 7 (2015)

29. A.I. Potekaev, A.M. Bubenchikov, M.A. Bubenchikov, Russ. Phys. J., 55, 12 (2013)

30. M.A. Bubenchikov, A.I. Potekaev, A.M. Bubenchikov, Russ. Phys. J., 56, 3 (2013)

31. M.A. Bubenchikov, A.I. Potekaev, A.M. Bubenchikov, Russ. Phys. J., 56, 7 (2013)

32. A.M. Bubenchikov, M.A. Bubenchikov, A.I. Potekaev, A.S. Maslov, V.V. Ovcharenko, O.V. Usenko, Russ. Phys. J., 57, 7 (2014)

33. M.A. Bubenchikov, A.I. Potekaev, A.M. Bubenchikov, Appl. Mech. Mater., 698 (2015)

34. M.A. Bubenchikov, A.M. Bubenchikov, O.V. Usenko, A.V. Ukolov, IOP CONF. SER. MATER. SCI. ENG, 87, 12015

35. A.M. Bubenchikov, M.A. Bubenchikov, A.I. Potekaev, E.E. Libin and Y.P. Khudobina, Russ.

Phys. J., 57, 8 (2014) 